\title{
Enhanced accuracy of kinetic information from CT-CPMG experiments by transverse rotating-frame spectroscopy
}

\author{
David Ban • Adam Mazur • Marta G. Carneiro - T. Michael Sabo • \\ Karin Giller • Leonardus M. I. Koharudin • Stefan Becker • \\ Angela M. Gronenborn $\cdot$ Christian Griesinger $\cdot$ Donghan Lee
}

Received: 26 April 2013/Accepted: 5 August 2013/Published online: 15 August 2013

(C) Springer Science+Business Media Dordrecht 2013

\begin{abstract}
Micro-to-millisecond motions of proteins transmit pivotal signals for protein function. A powerful technique for the measurement of these motions is nuclear magnetic resonance spectroscopy. One of the most widely used methodologies for this purpose is the constant-time Carr-Purcell-Meiboom-Gill (CT-CPMG) relaxation dispersion experiment where kinetic and structural information can be obtained at atomic resolution. Extraction of accurate kinetics determined from CT-CPMG data requires refocusing frequencies that are much larger than the nuclei's exchange rate between states. We investigated the effect when fast processes are probed by CT-CPMG experiments via simulation and show that if the intrinsic relaxation rate $\left(R_{2,0}^{C T-C P M G}\right)$ is not known a priori the extraction of accurate kinetics is hindered. Errors on the order of $50 \%$ in the exchange rate are attained when processes become fast, but are minimized to $5 \%$ with a priori $R_{2,0}^{C T-C P M G}$ information. To alleviate this
\end{abstract}

Electronic supplementary material The online version of this article (doi:10.1007/s10858-013-9769-z) contains supplementary material, which is available to authorized users.

D. Ban - A. Mazur - M. G. Carneiro - T. M. Sabo - K. Giller · S. Becker · C. Griesinger $(\bowtie) \cdot$ D. Lee $(\bowtie)$

Department for NMR-Based Structural Biology, Max-Planck Institute for Biophysical Chemistry, Am Fassberg 11,

37077 Göttingen, Germany

e-mail: cigr@nmr.mpibpc.mpg.de

D. Lee

e-mail: dole@nmr.mpibpc.mpg.de

L. M. I. Koharudin · A. M. Gronenborn

Department of Structural Biology, University of Pittsburgh

School of Medicine, 1050 Biomedical Science Tower 3,

3501 5th Ave, Pittsburgh, PA 15260, USA shortcoming, we developed an experimental scheme probing $R_{2,0}^{C T-C P M G}$ with large amplitude spin-lock fields, which specifically contains the intrinsic proton longitudinal Eigenrelaxation rate. Our approach was validated with ubiquitin and the Oscillatoria agardhii agglutinin (OAA). For OAA, an underestimation of $66 \%$ in the kinetic rates was observed if $R_{2,0}^{C T-C P M G}$ is not included during the analysis of CT-CPMG data and result in incorrect kinetics and imprecise amplitude information. This was overcome by combining CT-CPMG with $R_{2,0}^{C T-C P M G}$ measured with a high power $\mathrm{R}_{1 \rho}$ experiment. In addition, the measurement of $R_{2,0}^{C T-C P M G}$ removes the ambiguities in choosing between different models that describe CT-CPMG data.

Keywords Relaxation dispersion - CT-CPMG · $\mathrm{R}_{1 \rho} \cdot$ Kinetics

\section{Introduction}

Motions displayed by proteins on the micro-to-millisecond timescale are integral to their function. Within this timescale excursions in a ground-state ensemble (Ban et al. 2011; Lange et al. 2008) or to intermediates that are sparsely populated (Bhabha et al. 2011; Eisenmesser et al. 2005) have been linked to molecular recognition, or enzymatic catalysis, respectively. Nuclear magnetic resonance (NMR) is a key spectroscopic tool for investigating the different states of proteins. A particular unique phenomenon inherent to NMR is the ability to explore exchange. It is ascribed to events in which nuclei that interconvert between distinct chemical environments on the micro- to millisecond timescale contributes as a source of relaxation to a nuclei's effective transverse relaxation 
rate $\left(\mathrm{R}_{2, \mathrm{eff}}\right)$ (Neudecker et al. 2009; Palmer 2004; Palmer et al. 2001). The primary and most widely used NMR technique for probing $\mathrm{R}_{2, \text { eff }}$ is relaxation dispersion (RD) in which dispersion in $R_{2, \text { eff }}$ is created by altering the degree of refocusing for a given populated magnetization (Palmer et al. 2001). From such data, information about the interconversion rate $\left(\mathrm{k}_{\mathrm{ex}}\right)$ between the differently populated states, the difference in the chemical shifts of these states $(\Delta \omega)$, and the intrinsic relaxation rate $\left(\mathrm{R}_{2,0}\right)$ for a given nuclei can be extracted.

A severe drawback with the use of Carr-Purcell-Meiboom-Gill (CPMG) (Carr and Purcell 1954; Meiboom and Gill 1958) sequences as an RD experiment was that the applied frequency $\left(v_{\mathrm{CPMG}}\right)$ must be carefully considered to prevent generation of antiphase magnetization caused by scalar couplings and thus, only a short interpulse delay between $180^{\circ}$ pulses could be applied. This drawback could be alleviated by averaging the contributions to $\mathrm{R}_{2,0}$ from the inphase and the antiphase magnetization $\left(R_{2,0}^{C T-C P M G}\right)$ (Loria et al. 1999). This also allows for RD experiments to be carried out in a constant-time (Loria et al. 1999; Tollinger et al. 2001) (CT-CPMG) fashion, which dramatically reduces the measurement time (Skrynnikov et al. 2001). For the $\mathrm{RD}$ experiments using the CT-CPMG sequence, the applied frequency $\left(v_{\mathrm{CPMG}}\right)$ within a given constant-time block is varied in order to measure the change in $\mathrm{R}_{2 \text {,eff }}$ as a function of $v_{\mathrm{CPMG}}$. For increasing $v_{\mathrm{CPMG}}$, populated magnetization coherences become more completely refocused and $\mathrm{R}_{2 \text {,eff }}$ decreases to $R_{2,0}^{C T-C P M G}$, or the point at which all exchange has been removed. However, typical $v_{\text {CPMG }}$ values for ${ }^{15} \mathrm{~N}$ nuclei are limited to $1 \mathrm{kHz}$ (Ishima 2012). This constraint results in the inability to resolve fast kinetic processes since the exchange contribution that remains is not sampled and the overall exchange contribution $\left(R_{e x}=R_{2, \text { eff }}\left(v_{C P M G} \rightarrow 0\right)\right.$ $\left.-R_{2, e f f}\left(v_{C P M G} \rightarrow \infty\right)\right)$ will be underestimated. Here, we show that the extraction of kinetic information by CT-CPMG can be hindered when $R_{2,0}^{C T-C P M G}$ is not known a priori and included in the analysis, but can be alleviated by using large amplitude transverse-rotating frame spin-lock fields that are applied to same observables that are tracked during the CTCPMG experiments. The proposed experimental procedure outlined below is also a critical aspect since $R_{2,0}^{C T-C P M G}$ is not the same as what is obtained from standard ${ }^{15} \mathrm{~N}$ relaxation measurements (vide infra) (Farrow et al. 1994).

\section{Results and discussion}

Although the improvement of accuracy and precision in the CPMG fitting with a prior $R_{2,0}^{C T-C P M G}$ is known in the literature (Akke et al. 1998; Evenäs et al. 2001; Grey et al.
2003), the detail description of the improvement is still lacking and thus we employed Monte-Carlo (MC) simulations on synthetic data that were generated with various values of $\mathrm{k}_{\mathrm{ex}}$ using a simple two-state exchange scenario, $A \underset{k_{-1}}{\stackrel{k_{1}}{\rightleftarrows}} B \quad$ where $\quad \mathrm{k}_{1}+\mathrm{k}_{-1}=\mathrm{k}_{\mathrm{ex}}$, (see "Materials and Methods"; Fig. 1). These synthetic datasets were fitted to models described by the Bloch-McConnell equations (McConnell 1958) (BM; Fig. 1a, b), and the Luz-Meiboom equation (Luz and Meiboom 1963) (LM; Fig. 1c, d), with $R_{2,0}^{C T-C P M G}$ either as a parameter to be fitted (green circle in Fig. 1) or as a known parameter (blue circle in Fig. 1). It is apparent from Fig. 1 that the fractional error in the fitted exchange rate $\left(\mathrm{k}_{\mathrm{ex}}^{\mathrm{fit}}\right)$ relative to the true exchange rate $\left(\mathrm{k}_{\mathrm{ex}}^{\text {true }}\right.$ is the $\mathrm{k}_{\mathrm{ex}}$ value used as input for the generation of the synthetic sets) was as large as $\sim 30$ and $50 \%$ (in particular, when the exchange process is fast; $\mathrm{k}_{\mathrm{ex}}^{\text {true }} / \Delta \omega>\sim 4$ ) for the $\mathrm{BM}$ and LM models, respectively, when $R_{2,0}^{C T-C P M G}$ remained as a variable (green circles Fig. 1). In contrast, when $R_{2,0}^{C T-C P M G}$ was included as a known parameter during the minimization of a given synthetic set, the error in $\mathrm{k}_{\mathrm{ex}}^{\mathrm{fit}}$ does not exceed more than $5 \%$ over all ratios of $\mathrm{k}_{\mathrm{ex}}^{\mathrm{true}} / \Delta \omega$ in the simulations. The $R_{2,0}^{C T-C P M G}$ included in this analysis can be obtained from a different experiment, as suggested in this work. Further, the uncertainties of fitted $k_{\mathrm{ex}}(\sigma)$ were reduced by as much as $12-14$-fold when $R_{2,0}^{C T-C P M G}$ is known compared to the situation where $R_{2,0}^{C T-C P M G}$ has to be fitted (Fig. 1b, d). The pseudo Lorentzian profile from the reduction in $\left(\sigma_{R_{2,0}^{C T-C P M G}-\text { Known }}\right)$ beyond $\mathrm{k}_{\mathrm{ex}}^{\text {true }} / \Delta \omega>8$ (Fig. 1b, d) is due to the effect that as $\mathrm{k}_{\mathrm{ex}}^{\text {true }}$ increases the overall contribution of exchange $\left(\mathrm{R}_{\mathrm{ex}}=\mathrm{R}_{2, \mathrm{eff}}^{\text {(low } v \text { CPMG })}\right.$ $-\mathbf{R}_{2, \text { eff }}^{(\text {high } v \text { CPMG) }}$ ) decreases for those synthetic sets (Fig. S1) and introduces larger errors in $\mathrm{k}_{\mathrm{ex}}^{\mathrm{fit}}$. The simulations here were conducted with dispersion curves created at two different spectrometer field strengths as this is frequently done to increase the precision in the extracted parameters (Kovrigin et al. 2006). However, even with measurements conducted at a single field using a known $R_{2,0}^{C T-C P M G}$ during the fitting procedure still constitutes an advantage over leaving $R_{2,0}^{C T-C P M G}$ as an adjustable parameter (Fig. S2). Thus, it is clearly beneficial to directly measure $R_{2,0}^{C T-C P M G}$ and include this value as a known parameter for extracting accurate kinetic information from dispersion profiles. We also considered the situation in which $R_{2,0}^{C T-C P M G}$ is not directly fixed, but instead when a measurement is made with large $v_{\mathrm{CPMG}}$ values (Fig S3) (Akke et al. 1998; Evenäs et al. 2001; Grey et al. 2003). This produced a similar trend as in Fig. 1, but the accuracy in the determination at ratios of $\mathrm{k}_{\mathrm{ex}}^{\mathrm{true}} / \Delta \omega>10$ deteriorated as 


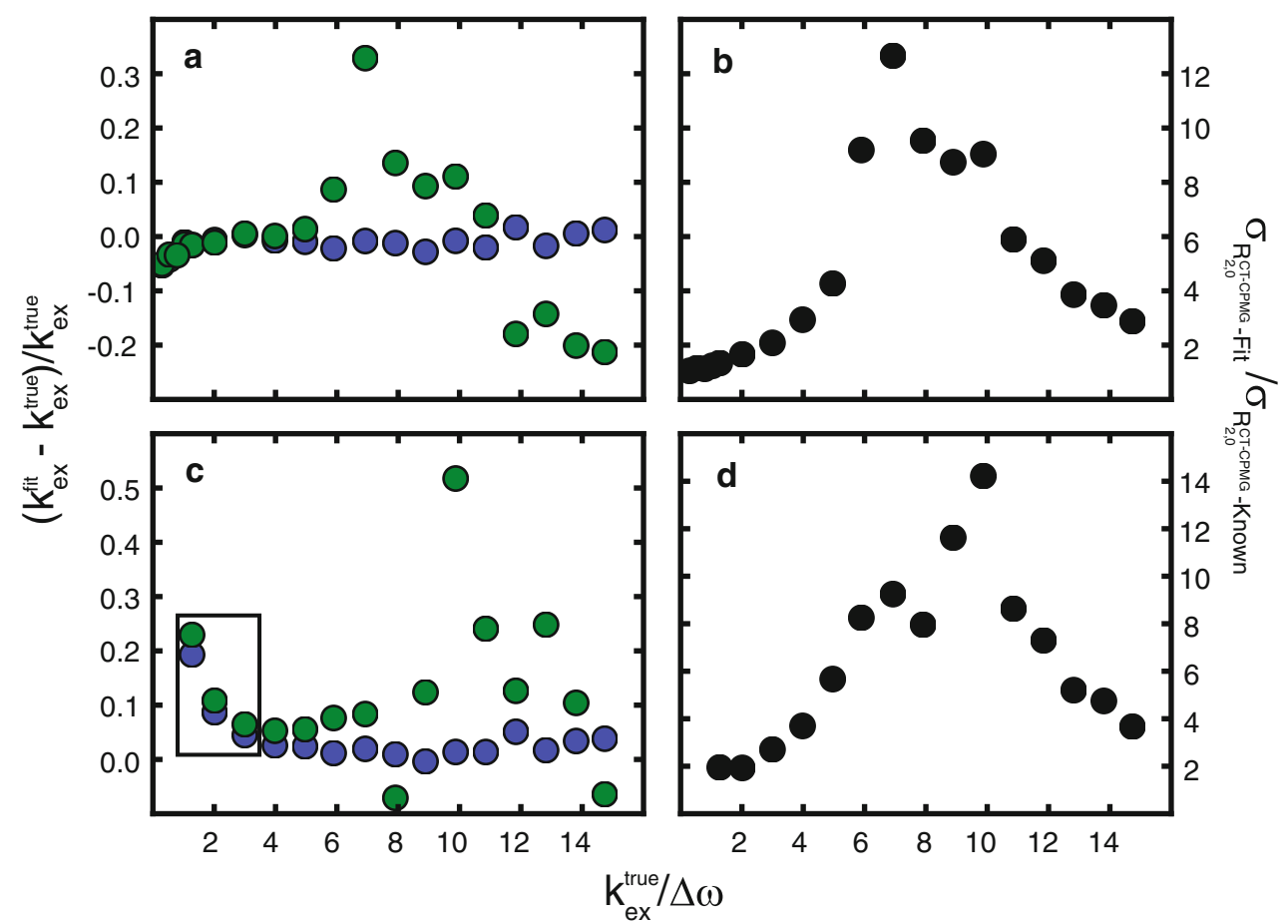

Fig. 1 a, c Comparison of the errors in the fitted exchange rate $\left(\mathrm{k}_{\mathrm{ex}}^{\mathrm{fit}}\right)$ as a fraction of the true exchange rate $\left(\mathrm{k}_{\mathrm{ex}}^{\text {true }}\right)$. Synthetic CT-CPMG data were generated up to a maximum $v_{\mathrm{CPMG}}$ of $1 \mathrm{kHz}$ (details in "Materials and Methods") and fitted to the Bloch-McConnell (BM; a and b) and the Luz-Meiboom (LM; c and d) equations with $R_{2,0}^{C T-C P M G}$ either as a fitting parameter (green circle in a and c) or a known parameter (blue circle in $\mathbf{a}$ and $\mathbf{c}$ ). b, d The ratio between

uncertainties in the determination of $\mathrm{k}_{\mathrm{ex}}^{\mathrm{fit}}$, defined as the standard deviation from Monte-Carlo (MC) runs using $R_{2,0}^{C T-C P M G}$ as a fitting parameter $\left(\sigma_{R_{2,0}^{C T-C P M G}-F i t}\right)$ and as a known parameter $\left(\sigma_{R_{2,0}^{C T-C P M G}-\text { Known }}\right)$. Overestimation of $\mathrm{k}_{\mathrm{ex}}^{\mathrm{fit}}$ in $\mathrm{c}$ for $1 \leq \mathrm{k}_{\mathrm{ex}}^{\mathrm{true}} / \Delta \omega<\sim 3$ due to the limitation of LM equation (Fig. S1) are boxed

compared to including $R_{2,0}^{C T-C P M G}$ as a known parameter (Fig S3).

In principle, the refocusing frequency $\left(v_{\mathrm{CPMG}}\right)$ used during a CT-CPMG experiment must be much larger than $\mathrm{k}_{\mathrm{ex}}$, to quench the contribution from exchange in the measured $\mathrm{R}_{2, \text { eff }}$ which then becomes $R_{2,0}^{C T-C P M G}$. However, the typical upper value of $v_{\text {CPMG }}$ that is utilized on conventional spectrometers, approximately $1 \mathrm{kHz}$ for ${ }^{15} \mathrm{~N}$, (Ishima 2012; Ishima and Torchia 2003) is not large enough to suppress exchange processes faster than $\sim 150 \mu$ s (Ishima and Torchia 1999; Palmer et al. 2001; Vallurupalli et al. 2011). Due to this restriction, high power transverse rotating frame relaxation (Palmer and Massi 2006) $\left(R_{1 \rho}\right)$ experiments can be used as an alternative for quenching exchange processes. Since the amplitude of the spin-lock fields $\left(v_{\mathrm{SL}}\right)$ in $\mathrm{R}_{1 \rho}$ experiments is larger than $v_{\text {CPMG }}$, more efficient quenching of a given exchange event is achieved and thus can provide a more accurate value of $R_{2,0}^{C T-C P M G}$. In $\mathrm{R}_{1 \rho}$ experiments, the fastest process that can be removed $\left(1 / 2 \pi v_{\mathrm{SL}}\right)$ depends on the largest amplitude of $v_{\mathrm{SL}}$ permitted. The recent development of high spin lock amplitudes using a cryogenically cooled probehead (Ban et al. 2012) is of keen interest since generated $v_{\mathrm{SL}}$ up to

$6 \mathrm{kHz}$ for ${ }^{15} \mathrm{~N}$ nuclei, which represent an improvement by a factor of three in the achievable field strength, can yield a $\mathrm{R}_{2, \text { eff }}$ that is free from an exchange process slower than $25 \mu \mathrm{s}$. Thus, we turn to develop the heteronuclear rotatingframe invasive nuclear exchange (HEROINE) experiment for measuring $\mathrm{R}_{2 \text {,eff }}$, utilizing the highest possible spin-lock field strength. The latter is included as currently the best measure of $R_{2,0}^{C T-C P M G}$ in order to improve the accuracy of model fitting data acquired from CT-CPMG experiments.

The pulse scheme of HEROINE (Fig. 2) monitors the same coherence as in CT-CPMG experiments (Loria et al. 1999; Tollinger et al. 2001), namely averaged inphase and antiphase $\left(1 / 2\left(\mathrm{~N}_{\mathrm{y}}+2 \mathrm{H}_{\mathrm{z}} \mathrm{N}_{\mathrm{x}}\right)\right)$ magnetization. Using INEPT, antiphase coherence $\left(2 \mathrm{H}_{\mathrm{Z}} \mathrm{N}_{\mathrm{x}}\right)$ is generated and its relaxation rate $\left(R_{1 \rho}^{a n t i}\right)$ is monitored by a spin-lock $\left(\mathrm{SL}_{\mathrm{x}}\right)$ with a time of $\mathrm{T} / 2$ $\left(I_{a} \propto \exp \left(-R_{1 \rho}^{a n t i} T / 2\right)\right)$. After point $a$ in Fig. 2, the antiphase coherence is transferred to an inphase coherence $\left(\mathrm{N}_{\mathrm{y}}\right)$, using a relaxation compensated element (Loria et al. 1999) and the relaxation rate of the inphase $\left(R_{1 \rho}^{i n}\right)$ is monitored by a spin-lock $\left(\mathrm{SL}_{\mathrm{y}}\right)$ for a time of $\mathrm{T} / 2$. Thus, at time point $\mathrm{b}$ in Fig. 2, the intensity is proportional to $\exp \left(-R_{1 \rho}^{a n t i} T / 2\right)$ 


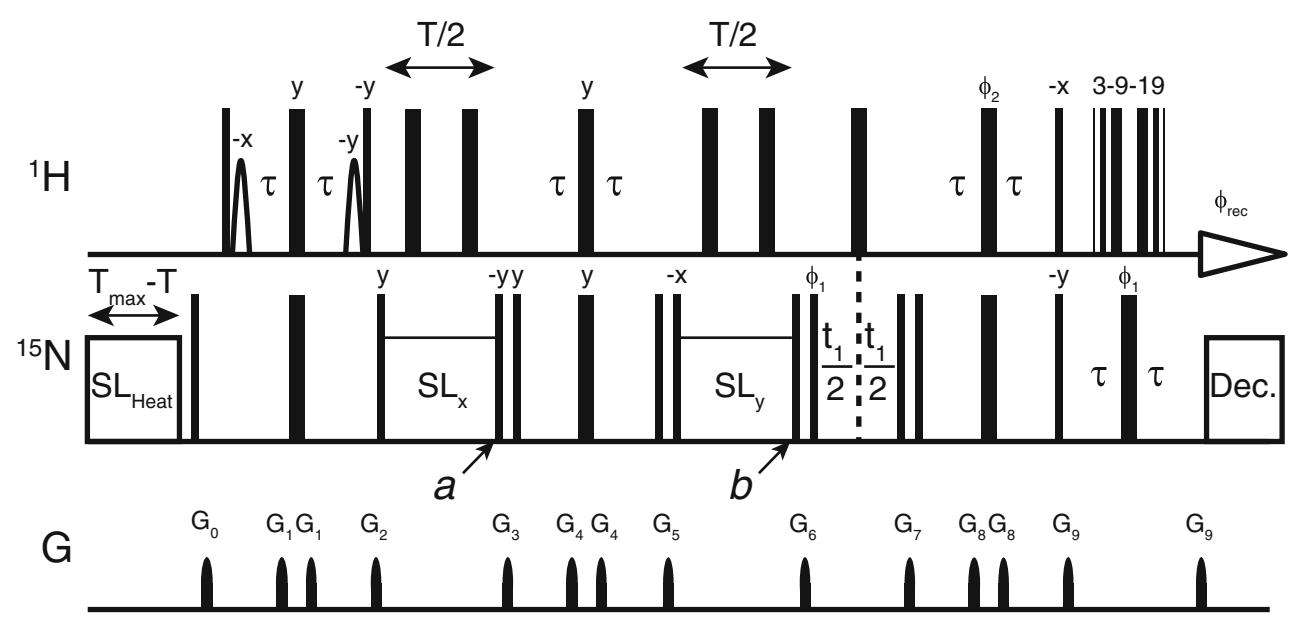

Fig. 2 Pulse scheme of the HEROINE experiment for measuring $\mathrm{R}_{2 \text {,eff }}$ utilizing a high spin-lock field strength, which can be used as $R_{2,0}^{C T-C P M G}$ for improving the accuracy of model fitting CPMG data. All ${ }^{1} \mathrm{H}$ and ${ }^{15} \mathrm{~N} 90^{\circ}$ and $180^{\circ}$ rectangular pulses are represented by narrow and wide black bars, respectively, and were applied at $35.7 \mathrm{kHz}$ and $10.6 \mathrm{kHz}$ for ${ }^{1} \mathrm{H}$ and ${ }^{15} \mathrm{~N}$, respectively. The shaped ${ }^{1} \mathrm{H}$ $90^{\circ}$ water selective pulses within the first INEPT block represent gaussian-shaped pulses and were applied for a duration of $1.5 \mathrm{~ms}$ in order to maintain water magnetization along the $+\mathrm{z}$ axis before the spin-lock period. Analogous to the relaxation compensated constant time CPMG (CT-CPMG) schemes (Loria et al. 1999; Tollinger et al. 2001), the relaxation delay (T) is split into two sections where $2 \mathrm{H}_{\mathrm{z}} \mathrm{N}_{\mathrm{x}}$ and $\mathrm{N}_{\mathrm{y}}$ coherences are each measured for a length of $\mathrm{T} / 2$ during the $\mathrm{SL}_{\mathrm{x}}$ and $\mathrm{SL}_{\mathrm{y}}$ spin-lock blocks, respectively. The field strength of the implemented SL field can be set to any value; here we used a large amplitude $\left(v_{\mathrm{SL}}=6 \mathrm{kHz}\right)$ to ensure that resonances which are onresonance had exchange processes with lifetimes up to $25 \mu \mathrm{s}$ quenched (Ban et al. 2012). Only resonances that are on-resonance are considered for further analysis. Therefore, a hard $90^{\circ}{ }^{15} \mathrm{~N}$ pulse suffices to place a target resonance within the transverse plane before the start of the spin-lock. Cross correlated relaxation between

$\exp \left(-R_{1 \rho}^{i n} T / 2\right)=\exp \left(-\left(\frac{R_{1 \rho}^{\text {anti }}+R_{1 \rho}^{i n}}{2}\right) T\right)$, compared with CTCPMG $\left(\exp \left(-\left(\frac{R_{2}^{\text {anti }}+R_{2}^{\text {in }}}{2}\right) T\right)=\exp \left(-R_{2}^{\text {eff }} T\right)\right)$. Since spinlock fields are used the exponential function takes the form $I(T)=I_{0} \exp \left(-R_{1 \rho}^{\text {eff }} T\right)$, where $R_{1 \rho}^{\text {eff }}$ is $\left(\frac{R_{1 \rho}^{\text {anti }}+R_{1 \rho}^{\text {in }}}{2}\right)$, and retains a rate of $R_{1 \rho}^{e f f}=R_{1} \cos ^{2} \theta+\left(R_{2,0}^{v S L \rightarrow \infty}+R_{1 \rho}^{e x}\right) \sin ^{2} \theta$ where $\mathrm{R}_{1}$, $R_{2,0}^{v S L \rightarrow \infty}, R_{1 \rho}^{e x}$, and $\theta$ are the longitudinal relaxation rate, the intrinsic transverse rotating-frame relaxation rate, contribution from exchange, and the tilt angle $\left(\theta=\tan ^{-1}\left(v_{\mathrm{SL}} / \Omega\right)\right.$; $\Omega=v_{0}-v_{\mathrm{RF}} ; v_{0}$ is the resonance frequency for a given nucleus and $v_{\mathrm{RF}}$ is where $v_{\mathrm{SL}}$ is applied), respectively. Here, $R_{2,0}^{v S L \rightarrow \infty}$ is again the average between inphase and antiphase relaxation rates measured by transverse rotating-frame experiments. For the CT-CPMG measured rate, $\mathrm{R}_{2, \text { eff }}$ can be expressed as $R_{2, e f f}=R_{2,0}^{C T-C P M G}+R_{C T-C P M G}^{e x}$. It is important to note that $R_{2,0}^{C T-C P M G}$ is the intrinsic exchange free relaxation rate for CT-CPMG experiments (Loria et al. 1999) and is not directly comparable to the pure inphase relaxation rate chemical shift anisotropy and dipolar coupling was suppressed by applying ${ }^{1} \mathrm{H} 180^{\circ}$ rectangular pulses at $\mathrm{T} / 8$ and $3 \mathrm{~T} / 8$ during a given SL period (Massi et al. 2004). To ensure equal power deposition during all values of $\mathrm{T}$, a linear temperature compensation scheme $\left(v_{\mathrm{SLheat}}=v_{\mathrm{SL}}\right)$ was used in which $\mathrm{SL}_{\mathrm{Heat}}$ is the difference between the longest relaxation delay $\left(\mathrm{T}_{\max }\right)$ and the length of a given total SL period (T). $\mathrm{SL}_{\text {Heat }}$ was applied at $28.4 \mathrm{kHz}$ upfield from the transmitter frequency. Before purging the ${ }^{15} \mathrm{~N}$ Boltzmann polarization, the ${ }^{15} \mathrm{~N}$ transmitter frequency was placed back on-resonance. During acquisition ${ }^{15} \mathrm{~N}$ decoupling was done with a WALTZ16 scheme at field strength of $2 \mathrm{kHz}$ (Shaka et al. 1983). The delay $\tau$ was set to $1 / 4 \mathrm{~J}_{\mathrm{NH}}(2.7 \mathrm{~ms})$. Frequency discrimination in the indirect dimension was achieved by States-TPPI (Marion et al. 1989) quadrature detection utilizing the following phase scheme: $\phi_{1}=(\mathrm{x}$, $-\mathrm{x},-\mathrm{x}, \mathrm{x}), \phi_{2}=(\mathrm{y}, \mathrm{y},-\mathrm{y},-\mathrm{y})$, and $\phi_{\mathrm{rec}}=(-\mathrm{x}, \mathrm{x}, \mathrm{x},-\mathrm{x})$. Phases for all pulses are $\mathrm{x}$ phase unless otherwise indicated. Gradients with strengths (length) of $\mathrm{G}_{0}=42 \mathrm{G} / \mathrm{cm}(1 \mathrm{~ms}), \mathrm{G}_{1}=10 \mathrm{G} / \mathrm{cm}(0.5 \mathrm{~ms})$, $\mathrm{G}_{2}=28 \mathrm{G} / \mathrm{cm}(0.5 \mathrm{~ms}), \mathrm{G}_{3}=38 \mathrm{G} / \mathrm{cm}(0.5 \mathrm{~ms}), \mathrm{G}_{4}=12 \mathrm{G} / \mathrm{cm}$ (0.5 ms), $\mathrm{G}_{5}=37 \mathrm{G} / \mathrm{cm}(0.5 \mathrm{~ms}), \mathrm{G}_{6}=16 \mathrm{G} / \mathrm{cm}(0.5 \mathrm{~ms}), \mathrm{G}_{7}=24$ $\mathrm{G} / \mathrm{cm}(0.5 \mathrm{~ms}), \mathrm{G}_{8}=20 \mathrm{G} / \mathrm{cm}(0.5 \mathrm{~ms})$, and $\mathrm{G}_{9}=35 \mathrm{G} / \mathrm{cm}(0.5 \mathrm{~ms})$ were used

measured by other relaxation techniques such as cross correlated relaxation (Farrow et al. 1994; Kroenke et al. 1998). In HEROINE, longitudinal proton relaxation is also sampled for a period of $T / 2$ due to the averaging between inphase and antiphase heteronuclear coherences. When HEROINE is performed on-resonance $\left(\theta=90^{\circ} ; R_{1}=0\right)$, the measured rate is $R_{1 \rho}^{\text {eff }}=R_{2,0}^{v S L \rightarrow \infty}+R_{1 \rho}^{e x}$. Therefore, if $v_{\mathrm{SL}}$ and $v_{\mathrm{CPMG}}$ in the HEROINE and CT-CPMG experiments, respectively, are sufficiently large to suppress the contribution of exchange on the relaxation rate $\left(R_{1 \rho}^{e x}-R_{C T-C P M G}^{e x}=0\right)$, the same intrinsic transverse relaxation rate will be achieved $\left(R_{2,0}^{C T-C P M G}=\right.$ $\left.R_{2,0}^{v S L \rightarrow \infty}\right)$. However, the maximum attenuation of a given exchange event monitored during a CT-CPMG experiment is often not sufficient, given the relatively small refocusing frequency, and may introduce an error in the extraction of kinetic information as described earlier. In contrast, using the recent development of applying a high spin-lock field strength up to $6 \mathrm{kHz}$ an exchange process slower than $25 \mu$ s can be 
quenched in HEROINE and a more veracious $R_{2,0}^{C T-C P M G}$ can be obtained (Ban et al. 2012). For the off-resonance $\left(\theta<90^{\circ}\right)$ situation, complications caused by a contribution from $R_{1}$ due to the tilt angle will arise. However, in the current implementation (using a $6 \mathrm{kHz}$ spin-lock field strength), only $2.2 \%$ contribution of $R_{1}$ is expected, even with an offset of $15 \mathrm{ppm}$ on a $600 \mathrm{MHz}$ spectrometer. Here, we repeated HEROINE at three different offsets and observed that $\mathrm{R}_{1}$ had a negligible contribution $(<0.1 \%)$ to $\mathrm{R}_{1 \rho}$.

In order to validate whether HEROINE results in the same $\mathrm{R}_{2 \text {,eff }}$ observed in CT-CPMG, we performed HEROINE and CT-CPMG measurements at $298 \mathrm{~K}$ on ${ }^{15} \mathrm{~N}$ labeled ubiquitin, since exchange slower than $25 \mu$ s would not contribute to the measurement of ${ }^{15} \mathrm{~N}$ relaxation (Ban et al. 2011; Massi et al. 2005). In Fig. 3a, typical HEROINE measurements for Ile23 and Lys39 are displayed showing that a monoexponential profile is retained and other effects from cross-correlated relaxation during the spin-lock period are re moved (Korzhnev et al. 2005; Massi et al. 2004). The correlation between $R_{2, \text { eff }}$ measured by CT-CPMG at a $v_{\text {CPMG }}$ value of $1 \mathrm{kHz}$ and HEROINE at a $v_{\mathrm{SL}}$ value of $6 \mathrm{kHz}$ is excellent with a Pearson correlation coefficient of 0.989 (Fig. 3b). The RMSD between the data points is $0.21 \mathrm{~s}^{-1}$ and is comparable to the average error derived from the CT-CPMG experiment rendering the rates from both experiments nearly indistinguishable. Thus, HEROINE probes the same $R_{2,0}^{C T-C P M G}$ as in CT-CPMG, and therefore this value can be used for the fitting procedure described earlier.

Approaches for the determination of the inphase intrinsic relaxation rates of ${ }^{15} \mathrm{~N}$ nuclei $\left(R_{2,0}^{N}\right)$ that differs from $R_{2,0}^{C T-C P M G}$ (Hansen et al. 2007; Wang et al. 2001) by the proton longitudinal Eigenrelaxation rate have been described in the literature, in which a series of experiments were used to measure only the contribution of dipoledipole relaxation (Hansen et al. 2007), or where measurements of heteronuclear-NOE, transverse and longitudinal cross-correlated relaxation (Kroenke et al. 1998; Millet et al. 2000) have been combined to calculate $R_{2,0}^{N}$. However, this $R_{2,0}^{N}$ cannot be used for the previously described procedure to reduce the error in the fitting because a quantification of $R_{2,0}^{N}$ does not reflect contributions from antiphase magnetization, of which the main relaxation mechanism is through remote protons and is a difficult contribution to quantify by only calculation (Lee et al. 2006; Long and Yang 2010; Loria et al. 1999). In principle, if the remote proton contribution could be measured separately, then $R_{2,0}^{C T-C P M G}$ could be calculated, but this may lead to a larger error in $R_{2,0}^{C T-C P M G}$ since uncertainties will be propagated from the different experiments. An
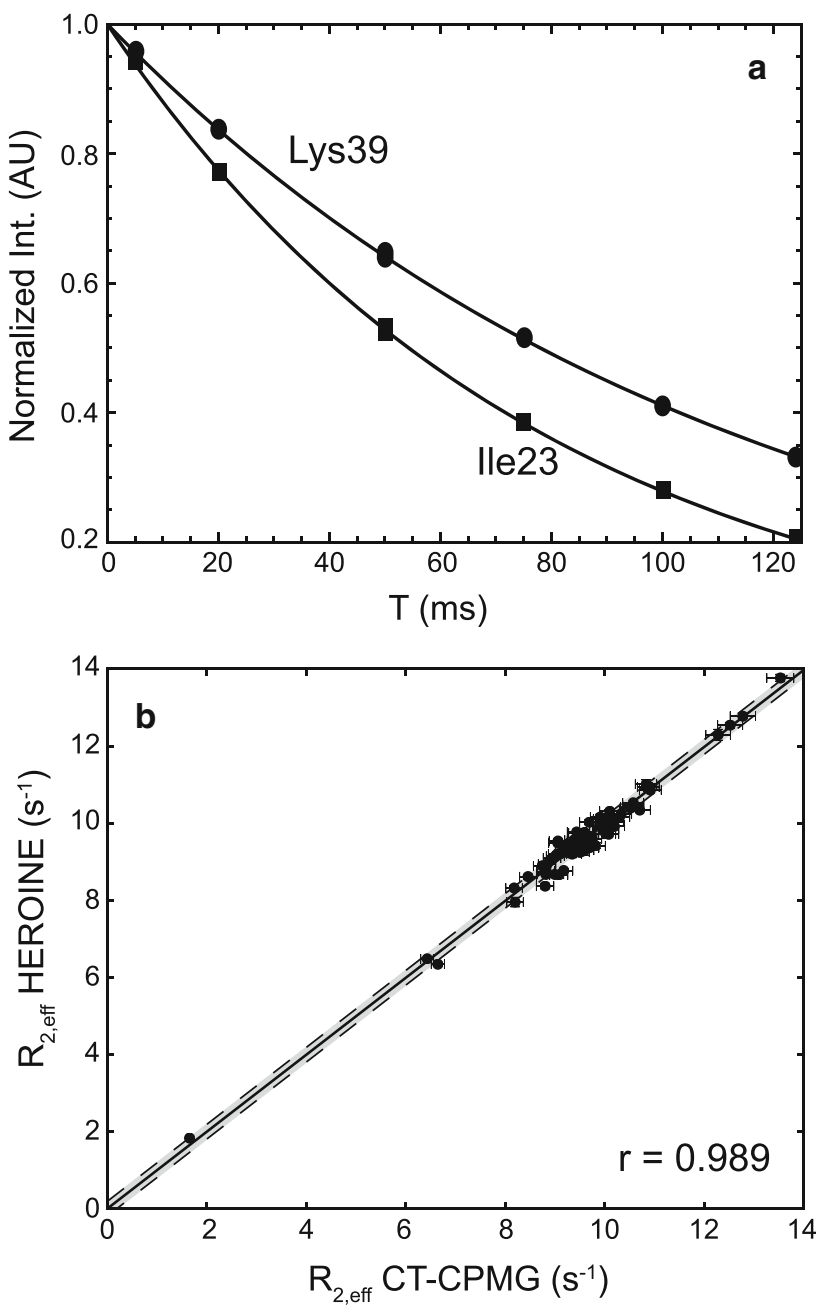

Fig. 3 Validation of HEROINE experiment using ${ }^{15} \mathrm{~N}$ labeled ubiquitin at $298 \mathrm{~K}$, at which exchange process slower than $20 \mu \mathrm{s}$

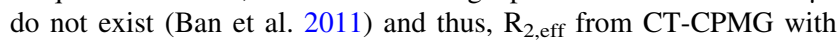
$1 \mathrm{kHz} \quad v_{\mathrm{CPMG}}$ and $6 \mathrm{kHz}$ HEROINE experiment should display similar values. a Example decay curves measured for residues Ile23 and Lys39 where points were sampled for 5-125 ms and fit to a monoexponential function. b Correlation between $\mathrm{R}_{2, \text { eff }}$ measured with HEROINE and CT-CPMG using $v_{\mathrm{SL}}$ and $v_{\mathrm{CPMG}}$ values at 6 and $1 \mathrm{kHz}$, respectively. The solid line in $\mathbf{b}$ has a slope of 0.998 and the Pearson correlation coefficient (r) is also given in $\mathbf{b}$ with a root mean square deviation of $0.21 \mathrm{~s}^{-1}$. The average error of the CT-CPMG experiment $\left(0.19 \mathrm{~s}^{-1}\right)$ is represented by the gray shading with the black dashed line boundaries in $\mathbf{b}$

alternative approach has also been reported where CTCPMG $R_{2, \text { eff }}$ is measured with a period where two spin order $\mathrm{R}_{2 \mathrm{HzNz}}$ is also recorded during the sequence (Wang et al. 2001), in order to make the derived $R_{2, \text { eff }}$ value approximately comparable to $R_{2,0}^{N}$. But only conventional values of $v_{\text {CPMG }}$ were considered which would not capture the exchange contribution for residues that are in the fastregime $\left(\tau_{\mathrm{ex}}<150 \mu \mathrm{s}\right)$. The motions in the fast-regime could be better sampled using combination of CPMG and 


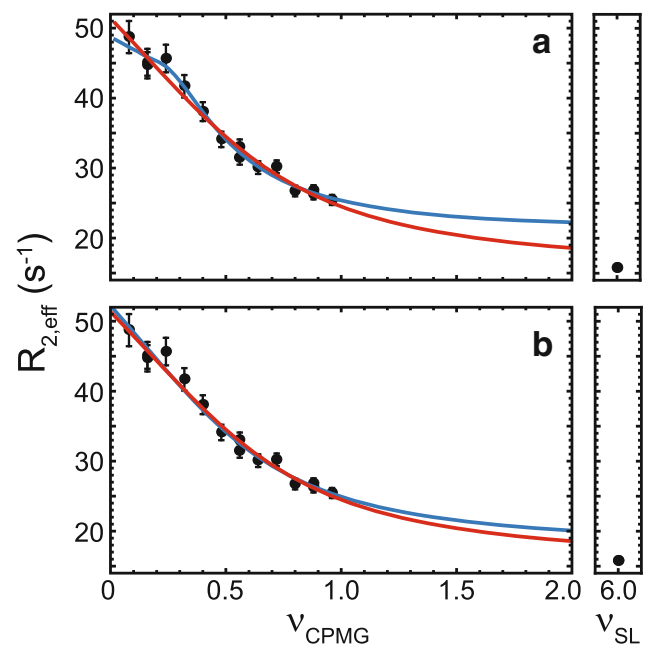

Fig. 4 Application of HEROINE to OAA. CT-CPMG dispersion curves were measured for W77 (a, b) and N99 (c, d) and display exchange on a fast timescale at $296 \mathrm{~K}$. For the CT-CPMG experiments on OAA, the maximum $v_{\mathrm{CPMG}}$ was $960 \mathrm{~Hz}$ and represents a conventional value used in CT-CPMG experiments (Ishima 2012). In $\mathbf{a}$ and $\mathbf{c}$ data were fit using the BM model while in $\mathbf{b}$ and $\mathbf{d}$ the LM model was applied. Fitted dispersion curves in blue correspond to minimizations where $R_{2,0}^{C T-C P M G}$ was a fitting parameter while red curves represent fits to curves using $\mathrm{R}_{2, \mathrm{eff}}$ from HEROINE as a known $R_{2,0}^{C T-C P M G}$. Strips next to the CT-CPMG data depict a given residue's measured $\mathrm{R}_{2, \mathrm{eff}}^{\mathrm{HEROINE}}$. Two residues were selected since they represent

$\mathrm{R}_{1 \rho}$ data (Evenäs et al. 2001). In contrast, HEROINE directly probes the actual magnetization that is used in CTCPMG experiments and is acquired via a single monoexponential decay profile as shown in Fig. 3 and uses spinlock fields where more efficient quenching of the exchange contribution can be attained.

As an example of a protein that exhibits exchange processes in the fast regime we selected the Oscillatoria aghardii agglutinin (OAA) (Koharudin et al. 2011; Koharudin and Gronenborn 2011), a lectin that binds high mannose glycans on GP120, a protein linked to the entry of HIV into human cells (Chan and Kim 1998). HEROINE together with CT-CPMG measurements were performed. From the CT-CPMG experiments performed on OAA at $296 \mathrm{~K}$, several residues in the carbohydrate binding pockets (Koharudin et al. 2011; Koharudin and Gronenborn 2011) undergo exchange. Among them, Trp77 and Asn99 exhibit the effect of a large (Fig. 4a, b) and small (Fig. 4c, d) underestimation in $\mathrm{R}_{\mathrm{ex}}$, respectively, for the CT-CPMG with $v_{\text {CPMG }}$ values up to $960 \mathrm{~Hz}$ compared to the HEROINE experiment that utilizes a $v_{\mathrm{SL}}$ with an amplitude of $6 \mathrm{kHz}$. Differences in $\mathrm{R}_{2, \text { eff }}\left(\delta \mathrm{R}_{2, \text { eff }}=\mathrm{R}_{2, \text { eff }}^{\mathrm{CT}-\mathrm{CPMG}}\right.$ $-\mathrm{R}_{2, \text { eff }}^{\mathrm{HEROINE}}$ ) for Trp77 and Asn99 are $9.67 \pm 1.07$ and $3.60 \pm 0.63 \mathrm{~s}^{-1}$, respectively, hamper the accuracy of the determined kinetic values (Table 1). For Trp77, if $R_{2,0}^{C T-C P M G}$ is

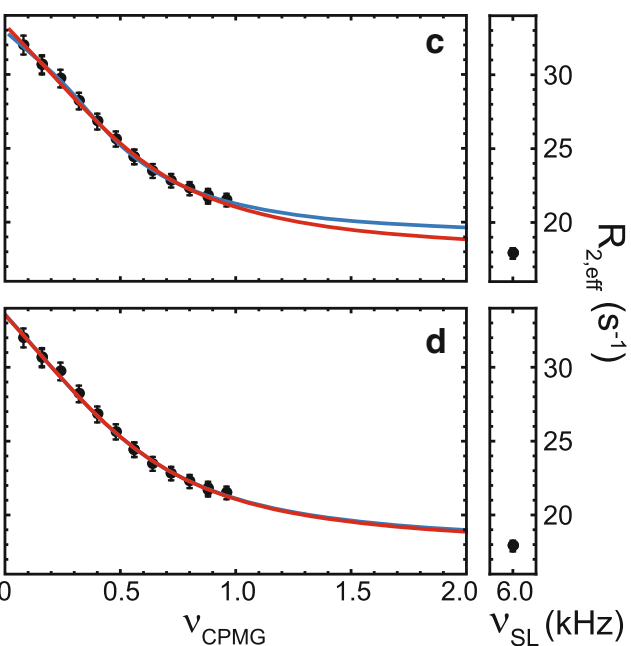

situations where fits without a priori knowledge of $R_{2,0}^{C T-C P M G}$ result in large (W77; a $66 \%$, b $12 \%$ ) and small (N99; c $34 \%$, d $2 \%$ ) deviations from the more accurately determined $\mathrm{k}_{\mathrm{ex}}$ when $R_{2,0}^{C T-C P M G}$ is known. Dispersion curves are plotted up to a $v_{\mathrm{CPMG}}$ value of $2 \mathrm{kHz}$ in order to readily detect the underestimation of the additional exchange contribution to $\mathrm{R}_{2, \mathrm{eff}}, \delta \mathrm{R}_{2, \mathrm{eff}}\left(\mathrm{R}_{2, \mathrm{eff}}^{\mathrm{CT}-\mathrm{CPMG}}-\mathrm{R}_{2, \mathrm{eff}}^{\mathrm{HEROINE}}\right)$. $\mathbf{a}, \mathbf{b} \delta \mathrm{R}_{2 \text {,eff }}$ for $\mathrm{W} 77$ is $9.67 \pm 1.07 \mathrm{~s}^{-1}$, and for $\mathrm{N} 99 \quad(\mathbf{c}, \quad \mathbf{d}) \quad \delta \mathrm{R}_{2 \text {,eff }}=$ $3.60 \pm 0.63 \mathrm{~s}^{-1}$. For a known $R_{2,0}^{C T-C P M G}$ a reduction in the errors of the fitted parameters is observed and convergence amongst the different models that are used to fit the data is reached (Table 1)

not fixed when fitting the data using the BM formalism, minimized values differ by $66 \%$ for $\mathrm{k}_{\mathrm{ex}}$, compared to a fit when $R_{2,0}^{C T-C P M G}$ is known from HEROINE. In addition, the chemical shift variance (Millet et al. 2000) $\left(\Phi=\mathrm{p}_{\mathrm{a}} \mathrm{p}_{\mathrm{b}} \Delta \omega^{2}\right)$ which is the product of the populations $\left(\mathrm{p}_{\mathrm{a}}\right.$ and $\left.\mathrm{p}_{\mathrm{b}}\right)$ and $\Delta \omega$, could not be determined with any precision. Such behavior, generally, would indicate that the BM model is inappropriate and instead the LM formalism should be used (Millet et al. 2000). When the LM model is used to fit the data with $R_{2,0}^{C T-C P M G}$ as an adjustable parameter, an even faster $\mathrm{k}_{\mathrm{ex}}$ is realized. More interestingly, as long as $R_{2,0}^{C T-C P M G}$ is kept fixed using the HEROINE measured value both BM and LM models converge to the same solution. In addition, as predicted from the simulations described above (Fig. 1), the error in $\mathrm{k}_{\mathrm{ex}}$ decreases by a factor of 6 and 2 for the BM and LM formalisms, respectively. Even for Asn99 (Fig. 4c, d) for which $\delta R_{2, \text { eff }}$ is smaller $\left(3.60 \pm 0.63 \mathrm{~s}^{-1}\right), \mathrm{k}_{\mathrm{ex}}$ determined from the $\mathrm{BM}$ approach while $R_{2,0}^{C T-C P M G}$ is kept as an adjustable parameter, within the error, gives a similar value like the other models. Again, the error associated with $\mathrm{k}_{\mathrm{ex}}$ using the $\mathrm{BM}$ and $\mathrm{LM}$ formalism is reduced by a factor of 2 and 5, respectively when the HEROINE measured $R_{2,0}^{C T-C P M G}$ is included in the analysis. These experimental results reported here are in accordance with the observations from the MC simulations in Fig. 1. 
Table 1 Results from fits to CT-CPMG data measured on OAA with and without the HEROINE determined $R_{2,0}^{C T-C P M G}$

\begin{tabular}{|c|c|c|c|c|}
\hline & $R_{2,0}^{C T-C P M G}-$ Fit BM & $R_{2,0}^{C T-C P M G}-$ Known BM & $R_{2,0}^{C T-C P M G}-$ Fit LM & $R_{2,0}^{C T-C P M G}-$ Known LM \\
\hline \multicolumn{5}{|l|}{ Trp77 } \\
\hline $\mathrm{k}_{\mathrm{ex}}\left(\mathrm{s}^{-1}\right)$ & $1,401 \pm 1,039$ & $4,095 \pm 160$ & $3,590 \pm 447$ & $4,078 \pm 160$ \\
\hline$\Phi \times 10^{3}\left(\mathrm{rad}^{2} \mathrm{~s}^{-2}\right)$ & $162 \pm 218$ & $145 \pm 10$ & $123 \pm 20$ & $145 \pm 3$ \\
\hline $\mathrm{AIC}_{\mathrm{c}}$ & 19.04 & 18.48 & 17.54 & 15.35 \\
\hline \multicolumn{5}{|l|}{ Asn99 } \\
\hline $\mathrm{k}_{\mathrm{ex}}\left(\mathrm{s}^{-1}\right)$ & $2,195 \pm 896$ & $3,329 \pm 478$ & $3,521 \pm 744$ & $3,591 \pm 157$ \\
\hline$\Phi \times 10^{3}\left(\operatorname{rad}^{2} \mathrm{~s}^{-2}\right)$ & $56.7 \pm 12.3$ & $58.5 \pm 5.6$ & $54.7 \pm 13.6$ & $56.2 \pm 1.4$ \\
\hline $\mathrm{AIC}_{\mathrm{c}}$ & 12.33 & 9.39 & 9.45 & 6.30 \\
\hline
\end{tabular}

The analysis of RD data also depends on selecting the correct model that describes the exchange dependence (Millet et al. 2000). Generally, newly acquired CT-CPMG data is initially fit to models that are valid over all timescales (Carver-Richards and BM) or the fast exchange LM model and then fit statistics $\left(\chi^{2}\right)$ are used in conjunction with $F$ tests to confirm the applicability of a given model (Skrynnikov et al. 2001). Instead, using HEROINE, a new protocol can be followed for handling data derived from CT-CPMG sequences. Once, $R_{2,0}^{C T-C P M G}$ is known, the BM and LM models converge to similar values, and therefore model selection becomes unnecessary. Thus, the BM model, which is valid over all timescales, can be used instead of the LM model. In particular, the HEROINE experiment may even provide the correct solution in the range where the $\mathrm{LM}$ model overestimates $\mathrm{k}_{\mathrm{ex}}\left(1 \leq \mathrm{k}_{\mathrm{ex}}^{\text {true }} /\right.$ $\Delta \omega<\sim 3$; square box in Fig. 1; Fig. S1) (Allerhand and Gutowsky 1964). Since the BM and LM models converge to similar solutions, the Akaike Information Criterion (Burnham and Anderson 2002) ( $\mathrm{AIC}_{\mathrm{c}}$, see "Materials and Methods") was used to identify the model that has the highest probability of best representing the current data set. In this case, for both Trp77 and Asn99 the LM model with $R_{2,0}^{C T-C P M G}$ determined from HEROINE (Table 1) was selected from the $\mathrm{AIC}_{\mathrm{c}}$ analysis.

\section{Conclusion}

We have demonstrated that if $R_{2,0}^{C T-C P M G}$ is known experimentally and therefore can be fixed during the analysis of CT-CPMG data, accurate kinetic parameters can be obtained over a broad range of $k_{\mathrm{ex}} / \Delta \omega$ ratios (Fig. 1). In order to measure an accurate $R_{2,0}^{C T-C P M G}$ valid for CTCPMG data, HEROINE was developed and validated (Figs. 2, 3) and shown to give access to this exchange free relaxation parameter in an unprecedentedly straightforward manner. HEROINE utilized large $v_{\mathrm{SL}}$ to measure an $\mathrm{R}_{2, \text { eff }}$ that represents a more true $R_{2,0}^{C T-C P M G}$ where motions up to
$25 \mu$ s are removed from $R_{2, \text { eff }}$. If $v_{\mathrm{SL}}$ values up to or exceeding $6 \mathrm{kHz}$ cannot be achieved with a given hardware configuration, it is possible to use lower $v_{\mathrm{SL}}$ values, although additional measurements at different offsets must be performed to maintain probed sites on-resonance. As shown for OAA, using the rate measured with HEROINE, as a way to constrain $R_{2,0}^{C T-C P M G}$, unifies different models that are frequently used to describe exchange (Table 1; Fig. 4). In addition, if HEROINE was not implemented for OAA, leaving $R_{2,0}^{C T-C P M G}$ as an adjustable fit parameter would have resulted in gross underestimation of the kinetics.

Including the transverse relaxation rate as a known parameter during the minimization procedures has beneficial effects, but depends on the exchange regime. For the slow exchange regime, or the case when $\Delta \omega$ is large compared to the exchange rate, two scenarios are possible. First, the exchange rate could be large enough that it is not quenched by employed CT-CPMG refocusing field strengths, but is still in the slow exchange regime due to a very large $\Delta \omega$. Second, the exchange rate is small enough to be quenched by the CPMG and $\Delta \omega$ remains significantly larger than the exchange rate. In our simulations, we could predict that the precision and the accuracy can be improved in both scenarios by including a measurement like HEROINE (data not shown, but for the latter case see Fig. 1). However, the origin of this improvement needs to be clarified. Particularly for the second scenario, the improvement in precision may be solely originated with excluding a fitting parameter, namely $R_{2,0}^{C T-C P M G}$. The effectiveness of the HEROINE experiment may also play a beneficiary role in scenarios where the kinetic scheme includes two processes, one that is slow and the other that is fast. The overlapping of two processes with distinct time scales can affect the fitted data given that using conventional refocusing frequencies may only sample the slower process. Thus, if only slow process is fitted on this data, the accuracy and the precision may be hampered. In this regard, the HEROINE experiment could be a tool to 
differentiate whether a system is simple two-state or more complicated scenarios. This effect is currently being investigated.

Fast regime motions measured by CT-CPMG experiments contain a parameter correlation (Fig. 4) between the populations and $\Delta \omega$. HEROINE, in combination with a recent approach (Vallurupalli et al. 2011) for breaking this correlation, using the difference in chemical shifts from single- and multiple quantum experiments, may further extend the limit at which populations and $\Delta \omega$ information can be extracted. HEROINE could also be adapted for the study of molecules with higher molecular weight. Even though HEROINE was demonstrated on relatively small systems, ubiquitin $(8.7 \mathrm{kDa})$ and OAA (14 kDa), this pulse sequence can be easily converted to incorporate a TROSY (Pervushin et al. 1997) readout for macromolecules of higher molecular weight. Additionally, if different nuclei are of interest the approach outlined above would still hold.

CT-CPMG experiments have come into widespread use for studying folding intermediates (Korzhnev et al. 2010), enzymatic catalysis (Bhabha et al. 2011; Eisenmesser et al. 2005), and protein-ligand interactions (Korzhnev et al. 2009; Sugase et al. 2007). Therefore, the inclusion of HEROINE with large $v_{\mathrm{SL}}$ derived $R_{2,0}^{C T-C P M G}$ for analyzing CT-CPMG data will augment the current methodology in order to improve the accuracy and reliability of kinetic data from the micro- to millisecond timescale.

\section{Materials and methods}

\section{Simulation}

The assessment of CT-CPMG data fitted with and without a known $R_{2,0}^{C T-C P M G}$ was performed on synthetic data sets created using the analytical Carver-Richards equation that is valid over all timescales (Carver and Richards 1972; Davis et al. 1994). Data was created for a single residue, with $\mathrm{k}_{\mathrm{ex}}$ values between 300 and 15,000 s $\mathrm{s}^{-1}$ (defined in Fig. 1 as $\mathrm{k}_{\mathrm{ex}}^{\text {true }}$ ), and $\Delta \omega, \mathrm{p}_{\mathrm{b}}$ (the minor population), and $R_{2,0}^{C T-C P M G}$ were kept constant at $2 \mathrm{ppm}, 0.05$, and $10 \mathrm{~s}^{-1}$, respectively. For each exchange scenario, 100 dispersion curves were created at two fields, 60.8 and $81 \mathrm{MHz}$ for

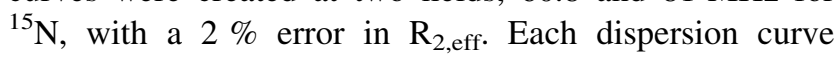
consisted of seventeen points and $v_{\mathrm{CPMG}}$ varied between 40 and $1,000 \mathrm{~Hz}$. Each set was fit either with the BM (McConnell 1958) or LM (Meiboom and Gill 1958) formalism using a non-linear Least Squares algorithm (see the Supplementary Information for the equations used in the fitting routine). From which, $\mathrm{k}_{\mathrm{ex}}^{\mathrm{fit}}$ was defined as the average $\mathrm{k}_{\mathrm{ex}}$, and $\sigma$ was the standard deviation in $\mathrm{k}_{\mathrm{ex}}$ of all minimizations in a given synthetic set. For the BM model, synthetic sets were used in which the ratio of $\mathrm{k}_{\mathrm{ex}}^{\text {true }} / \Delta \omega<1$ $\mathrm{R}_{2 \text {,eff }}$ was calculated only over the magnetization of the major state $\left(\mathrm{p}_{\mathrm{a}}\right)\left(R_{2, \text { eff }}=-1 / T \ln \left(M_{a}(t) / M_{a}(0)\right)\right)$, whereas for $\mathrm{k}_{\mathrm{ex}}^{\text {true }} / \Delta \omega \geq 1 \mathrm{R}_{2 \text {,eff }}$ was summed over the major and minor state $\left(R_{2, e f f}=-1 / T \ln \left(M_{a}(t)+M_{b}(t)\right)\right)$ (Myint and Ishima 2009). The LM model was only applied for sets for which $\mathrm{k}_{\mathrm{ex}}^{\text {true }} / \Delta \omega>1$ (Allerhand and Gutowsky 1964; Luz and Meiboom 1963).

\section{NMR spectroscopy}

Samples contained $2 \mathrm{mM}{ }^{15} \mathrm{~N}$ labeled ubiquitin, in $50 \mathrm{mM}$ sodium phosphate buffer, $\mathrm{pH} 6.5,100 \mathrm{mM} \mathrm{NaCl}, 0.05 \%$ $\mathrm{NaN}_{3}, 5 \% \mathrm{D}_{2} \mathrm{O} / 95 \% \mathrm{H}_{2} \mathrm{O}$ or $1 \mathrm{mM}{ }^{15} \mathrm{~N}$ labeled OAA in $20 \mathrm{mM}$ sodium acetate buffer, $\mathrm{pH} 5,20 \mathrm{mM} \mathrm{NaCl}, 3 \mathrm{mM}$ $\mathrm{NaN}_{3}, 5 \% \mathrm{D}_{2} \mathrm{O} / 95 \% \mathrm{H}_{2} \mathrm{O}$. CT-CPMG experiments followed the scheme of Long et al. (Long and Yang 2010; Pervushin et al. 1997). The phases of the ${ }^{15} \mathrm{~N}$ pulses during the CT-CPMG blocks were alternated in order to minimize off-resonance effects (Yip and Zuiderweg 2004). The pulse schematic for the CT-CPMG experiment used here can be found in the Supplementary Information (Figure S4). Constant relaxation times of 60 and $50 \mathrm{~ms}$ were used, and $v_{\mathrm{CPMG}}$ values from 67 to $1,000 \mathrm{~Hz}$ and 80 to $960 \mathrm{~Hz}$ were employed for ubiquitn and OAA, respectively. Three duplicates per dispersion profile were used for error estimation in $\mathrm{R}_{2 \text {,eff }}$ (Korzhnev et al. 2004). In total $80\left(\mathrm{t}_{1, \max }=41.1 \mathrm{~ms}\right.$ ) and $512\left(\mathrm{t}_{2, \max }=61 \mathrm{~ms}\right)$ complex points in the indirect and direct dimensions, respectively, were collected with 8 transients per point for ubiquitin. For OAA, 100 $\left(\mathrm{t}_{1, \max }=48.4 \mathrm{~ms}\right)$ and $512\left(\mathrm{t}_{2, \max }=61 \mathrm{~ms}\right)$ complex points in the indirect and direct dimensions, respectively, were acquired with 32 transients per point. Recycle delays of 2 and $1.5 \mathrm{~s}$ were used for ubiquitin and OAA, respectively. All spectra were processed and peak amplitudes determined using the NMRPipe software package (Delaglio et al. 1995). Analysis of CT-CPMG data followed procedures set in the literature (Korzhnev et al. 2004, 2009). We utilized $\mathrm{AIC}_{\mathrm{c}}$ since the compared models are not nested (Burnham and Anderson 2002). $\quad \mathrm{AIC}_{\mathrm{c}}$ was calculated as $\chi^{2}+$ $2 m\left(1+\frac{m+1}{1-m-1}\right)$ where $\chi^{2}$ is the target function value from a Least-Sqaures minimization, $\mathrm{m}$ the number of parameters in a given model, and 1 the total number of $R_{2, \text { eff }}$ values $(1=15)$ (Burnham and Anderson 2002).

Details in the setup of HEROINE are provided in the caption of Fig. 2. Only residues that are on-resonance with the applied $v_{\mathrm{SL}}$ are considered for analysis. Analysis of resonances is facilitated by using the largest $v_{\mathrm{SL}}$ possible in order to eliminate $\mathrm{R}_{1}$ and offset effects. HEROINE was measured at three different offsets $(\Omega)$, reducing the maximum $\mathrm{R}_{1}$ contribution to $\mathrm{R}_{1 \rho}$ to $0.1 \%$. The application and calibration for a $v_{\mathrm{SL}}$ strength of $6 \mathrm{kHz}$ was carried out as 
described in reference 20. Decay profiles were recorded by varying the relaxation period, $\mathrm{T}$, between $5-125 \mathrm{~ms}$ and 5-100 ms for ubiquitin and OAA, respectively. These profiles were subsequently fit to a monoexponential function as described above. For ubiquitin, spectra were collected with 8 scans per point, for a total of $80\left(\mathrm{t}_{1, \max }=41.1 \mathrm{~ms}\right)$ and $512\left(\mathrm{t}_{2, \max }=61 \mathrm{~ms}\right)$ complex points in the indirect, and direction dimensions, respectively. For OAA, spectra were acquired with $90 \quad\left(\mathrm{t}_{1, \max }=43.5 \mathrm{~ms}\right)$ and 512 $\left(\mathrm{t}_{2, \max }=61 \mathrm{~ms}\right)$ complex points in the indirect and direct dimensions, respectively with 8 transients per point. The recycle delay used in experiments with ubiquitin and OAA were 3 and $2 \mathrm{~s}$, respectively. In order to match the temperatures between both CT-CPMG and HEROINE experiments a temperature compensation scheme (Wang and Bax 1993) was employed depending on the recycle delays, relaxation delays, $v_{\mathrm{CPMG}}$ and $v_{\mathrm{SL}}$ from both experiments. Details can be found in the Supplementary Information (Figure S4). This temperature regulation scheme maintained that both experiments were conducted under equal temperatures, which allows for the Variable Temperature Unit to be offset to the desired temperature. For ubiquitin and OAA on increase of 0.7 and $0.9 \mathrm{~K}$, respectively was observed when a $v_{\mathrm{SL}}$ of $6 \mathrm{kHz}$ was applied. The coil temperature did not deviate during the application of the ${ }^{15} \mathrm{~N} 6 \mathrm{kHz}$ field strength (Fig. S5). Acquisition of all NMR data was on a Bruker Avance I spectrometer operating at a ${ }^{1} \mathrm{H}$ Larmor frequency of $600 \mathrm{MHz}$ with a QCI cryoprobe. The pulse sequences used here can also be found in the Bruker library of pulse programs. The analysis of CT-CPMG data with HEROINE derived $R_{2,0}^{C T-C P M G}$ values has also been incorporated in the ShereKahn program (Mazur et al. 2013) which can be found at http://sherekhan.bionmr.org.

Acknowledgments The work was supported by the Max Planck Society and the EU (ERC Grant Agreement Number 233227) (both to C.G.), and an NIH Grant to A.M.G (GM080642). We are grateful to Dr. Wolfgang Bermel and to Dr. Daniel Mathieu, Bruker Karlsruhe, for uploading the experimental schemes used here to the Bruker standard library (hsqcrexf3gpphtc193d).

\section{References}

Akke M, Liu J, Cavanagh J, Erickson HP, Palmer AG 3rd (1998) Pervasive conformational fluctuations on microsecond time scales in a fibronectin type III domain. Nat Struct Biol 5:55-59

Allerhand A, Gutowsky HS (1964) Spin-echo studies of chemical exchange II. Closed formulas for two sites. J Chem Phys 42:1587-1599

Ban D et al (2011) Kinetics of conformational sampling in ubiquitin. Angew Chem Int Ed Engl 50:11437-11440

Ban D et al (2012) Exceeding the limit of dynamics studies on biomolecules using high spin-lock field strengths with a cryogenically cooled probehead. J Magn Reson 221:1-4
Bhabha $G$ et al (2011) A dynamic knockout reveals that conformational fluctuations influence the chemical step of enzyme catalysis. Science 332:234-238

Burnham KP, Anderson DR (2002) Model selection and multi-model inference. Springer, Berlin

Carr HY, Purcell EM (1954) Effects of diffusion on free precession in nuclear magnetic resonance experiments. Phys Rev 54:630-638

Carver JP, Richards RE (1972) A general two-site solution for the chemical exchange produced dependence of $\mathrm{T}_{2}$ upon the CarrPurcell pulse separation. J Magn Reson 6:89-105

Chan DC, Kim PS (1998) HIV entry and its inhibition. Cell 93:681-684

Davis DG, Perlman ME, London RE (1994) Direct measurements of the dissociation-rate constant for inhibitor-enzyme complexes via the $\mathrm{T}_{1 \rho}$ and $\mathrm{T}_{2}$ (CPMG) methods. J Magn Reson 104:266-275

Delaglio $F$ et al (1995) NMRPipe: a multidimensional spectral processing system based on UNIX pipes. J Biomol NMR 6:277-293

Eisenmesser EZ et al (2005) Intrinsic dynamics of an enzyme underlies catalysis. Nature 438:117-121

Evenäs J, Malmendal A, Akke M (2001) Dynamics of the transition between open and closed conformations in a calmodulin C-terminal domain mutant. Structure 9:185-195

Farrow NA et al (1994) Backbone dynamics of a free and phosphopeptide-complexed Src homology 2 domain studied by ${ }^{15} \mathrm{~N}$ NMR relaxation. Biochemistry 33:5984-6003

Grey MJ, Wang C, Palmer AG 3rd (2003) Disulfide bond isomerization in basic pancreatic trypsin inhiptor: multisite chemical exchange quantified by CPMG relaxation dispersion and chemical shift modeling. J Am Chem Soc 125:14324-14335

Hansen DF et al (2007) An exchange-free measure of ${ }^{15} \mathrm{~N}$ transverse relaxation: an NMR spectroscopy application to the study of a folding intermediate with pervasive chemical exchange. J Am Chem Soc 129:11468-11479

Ishima R (2012) Recent developments in ${ }^{15} \mathrm{~N}$ NMR relaxation studies that probe protein backbone dynamics. Top Curr Chem 326:99-122

Ishima R, Torchia DA (1999) Estimating the time scale of chemical exchange of proteins from measurements of transverse relaxation rates in solution. J Biomol NMR 14:369-372

Ishima R, Torchia DA (2003) Extending the range of amide proton relaxation dispersion experiments in proteins using a constanttime relaxation-compensated CPMG approach. J Biomol NMR 25:243-248

Koharudin LM, Gronenborn AM (2011) Structural basis of the antiHIV activity of the cyanobacterial Oscillatoria agardhii agglutinin. Structure 19:1170-1181

Koharudin LM, Furey W, Gronenborn AM (2011) Novel fold and carbohydrate specificity of the potent anti-HIV cyanobacterial lectin from Oscillatoria agardhii. J Biol Chem 286:1588-1597

Korzhnev DM et al (2004) Low-populated folding intermediates of Fyn SH3 characterized by relaxation dispersion NMR. Nature 430:586-590

Korzhnev DM, Orekhov VY, Kay LE (2005) Off-resonance $\mathrm{R}_{1 \rho}$ NMR studies of exchange dynamics in proteins with low spin-lock fields: an application to a Fyn SH3 domain. J Am Chem Soc 127:713-721

Korzhnev DM, Bezsonova I, Lee S, Chalikian TV, Kay LE (2009) Alternate binding modes for a ubiquitin-SH3 domain interaction studied by NMR spectroscopy. J Mol Biol 386:391-405

Korzhnev DM, Religa TL, Banachewicz W, Fersht AR, Kay LE (2010) A transient and low-populated protein-folding intermediate at atomic resolution. Science 329:1312-1316

Kovrigin EL, Kempf JG, Grey MJ, Loria JP (2006) Faithful estimation of dynamics parameters from CPMG relaxation dispersion measurements. J Magn Reson 180:93-104 
Kroenke CD, Loria JP, Lee LK, Rance M, Palmer AG 3rd (1998) Longitudinal and transverse ${ }^{1} \mathrm{H}-{ }^{15} \mathrm{~N}$ Dipolar $/{ }^{15} \mathrm{~N}$ chemical shift anisotropy relaxation interference: unambiguous determination of rotational diffusion tensors and chemical exchange effects in biological macromolecules. J Am Chem Soc 120:7905-7915

Lange OF et al (2008) Recognition dynamics up to microseconds revealed from an RDC-derived ubiquitin ensemble in solution. Science 320:1471-1475

Lee D, Hilty C, Wider G, Wüthrich K (2006) Effective rotational correlation times of proteins from NMR relaxation interference. J Magn Reson 178:72-76

Long D, Yang D (2010) Millisecond timescale dynamics of human liver fatty acid binding protein: testing of its relevance to the ligand entry process. Biophys J 98:3054-3061

Loria JP, Rance M, Palmer AG 3rd (1999) A relaxation-compensated Carr-Purcell-Meiboom-Gill sequence for characterizing chemical exchange by NMR spectroscopy. J Am Chem Soc 121:2331-2332

Luz Z, Meiboom S (1963) Nuclear magnetic resonance study of the protolysis of trimethylammonium ion in aqueous solution-order of the reaction with respect to solvent. J Chem Phys 39:366-370

Marion D, Ikura M, Tschudin R, Bax A (1989) Rapid recording of 2D NMR spectra without phase cycling. Application to the study of hydrogen exchange in proteins. J Magn Reson 85:393-399

Massi F, Johnson E, Wang C, Rance M, Palmer AG 3rd (2004) NMR $\mathrm{R}_{1 \rho}$ rotating-frame relaxatin with weak radio-frequency fields. J Am Chem Soc 126:2247-2256

Massi F, Grey MJ, Palmer AG 3rd (2005) Microsecond timescale backbone conformational dynamics in ubiquitin studied with NMR $R_{1 \rho}$ relaxation experiments. Prot Sci 14:735-742

Mazur A, Hammesfahr B, Griesinger C, Kollmar M (2013) ShereKhan-calculating exchange parameters in relaxation dispersion data from CPMG experiments. Bioinformatics 29:1819-1820

McConnell HM (1958) Reaction rates by nuclear magnetic resonance. J Chem Phys 28:430-431

Meiboom S, Gill S (1958) Modified spin-echo method for measuring nuclear relaxation times. Rev Sci Inst 29:688-691

Millet O, Loria JP, Kroenke CD, Pons M, Palmer AG 3rd (2000) The static magnetic field dependence of chemical exchange linebroadening defines the NMR chemical shift time scale. J Am Chem Soc 122:2867-2877

Myint W, Ishima R (2009) Chemical exchange effects during refocusing pulses in constant-time CPMG relaxation dispersion experiments. J Biomol NMR 45:207-216
Neudecker P, Lundstrom P, Kay LE (2009) Relaxation dispersion NMR spectroscopy as a tool for detailed studies of protein folding. Biophys J 96:2045-2054

Palmer AG 3rd (2004) NMR characterization of the dynamics of biomacromolecules. Chem Rev 104:3623-3640

Palmer AG 3rd, Massi F (2006) Characterization of the dynamics of biomacromolecules using rotating-frame spin relaxation NMR spectroscopy. Chem Rev 106:1700-1719

Palmer AG 3rd, Kroenke CD, Loria JP (2001) Nuclear magnetic resonance methods for quantifying microsecond-to-millisecond motions in biological macromolecules. Methods Enzymol 339:204-238

Pervushin K, Riek R, Wider G, Wüthrich K (1997) Attenuated $T_{2}$ relaxation by mutual cancellation of dipole-dipole coupling and chemical shift anisotropy indicates an avenue to NMR structures of very large biological macromolecules in solution. Proc Natl Acad Sci USA 94:12366-12371

Shaka AJ, Keeler J, Frenkiel T, Freeman R (1983) An improved sequence for broadband decoupling: WALTZ-16. J Magn Reson $52: 335-338$

Skrynnikov NR, Mulder FA, Hon B, Dahlquist FW, Kay LE (2001) Probing slow time scale dynamics at methyl-containing side chains in proteins by relaxation dispersion NMR measurements: application to methionine residues in a cavity mutant of $\mathrm{T} 4$ lysozyme. J Am Chem Soc 123:4556-4566

Sugase K, Dyson HJ, Wright PE (2007) Mechanism of coupled folding and binding of an intrinsically disordered protein. Nature 447:1021-1025

Tollinger M, Skrynnikov NR, Mulder FA, Forman-Kay JD, Kay LE (2001) Slow dynamics in folded and unfolded states of an SH3 domain. J Am Chem Soc 123:11341-11352

Vallurupalli P, Bouvignies G, Kay LE (2011) Increasing the exchange time-scale that can be probed by CPMG relaxation dispersion NMR. J Phys Chem B 115:14891-14900

Wang AC, Bax A (1993) Minimizing the effects of radio-frequency heating in multidimensional NMR experiments. J Biomol NMR 3:715-720

Wang C, Grey MJ, Palmer AG 3rd (2001) CPMG sequences with enhanced sensitivity to chemical exchange. J Biomol NMR 21:361-366

Yip GN, Zuiderweg ER (2004) A phase cycle scheme that significantly suppresses offset-dependent artifacts in the $\mathrm{R}_{2}-\mathrm{CPMG}{ }^{15} \mathrm{~N}$ relaxation experiment. J Magn Reson 171:25-36 